\title{
LIPSCHITZ CLASSES OF SOLUTIONS TO CERTAIN ELLIPTIC EQUATIONS
}

\author{
Craig A. Nolder \\ The Florida State University, Department of Mathematics B-154 \\ Tallahassee, FL 32306-3027, U.S.A.
}

\begin{abstract}
We consider weak solutions $u$ in the Sobolev space $W_{\alpha, \text { loc }}^{1}(\Omega), \Omega \subset \mathbf{R}^{n}$, to elliptic equations of the form $\operatorname{div} A(x, \nabla u)=B(x, \nabla u)$. The Lipschitz continuity of $u$ over $\Omega$ is characterized by the growth of the local $L^{\alpha}$-average of $\nabla u$. Previous results cover the case in which the structure exponent $\alpha \geq n$. We give here a proof valid for all $1<\alpha<\infty$.
\end{abstract}

\section{Introduction and main results}

Theorem 1.1 follows from results in [HL]. Throughout $\Omega$ will be a connected open subset of $\mathbf{R}^{n}$. When $f: \Omega \rightarrow \mathbf{R}^{m}, 0<k \leq 1$, we write

$$
\|f\|^{k}=\sup _{\substack{x_{1}, x_{2} \in \Omega \\ x_{1} \neq x_{2}}}\left|f\left(x_{1}\right)-f\left(x_{2}\right)\right| /\left|x_{1}-x_{2}\right|^{k} .
$$

Theorem 1.1. Let $u$ be harmonic in the unit disk $D \subset \mathbf{R}^{2}$ and $0<k \leq 1$. If there exists a constant $C_{1}$ such that

$$
|\nabla u(z)| \leq C_{1}(1-|z|)^{k-1}
$$

for all $z \in D$, then there exists a constant $C_{2}$, depending only on $\alpha$ and $C_{1}$, such that

$$
\|u\|^{k} \leq C_{2} .
$$

Conversely, (1.3) implies that (1.2) holds for all $z \in D$ with $C_{1}$ depending only on $\alpha$ and $C_{2}$.

An analogue of Theorem 1.1 is given in [N2] for solutions of certain elliptic equations in divergence form. The main result of this paper, Theorem 1.11, extends this analogue to a larger class of such equations and other domains in $\mathbf{R}^{n}$.

More precisely, we consider weak solutions $u$ to equations of the form

$$
\operatorname{div} A(x, \nabla u)=B(x, \nabla u)
$$

1991 Mathematics Subject Classification: Primary 35J65; Secondary 30C65. 
in domains $\Omega \subset \mathbf{R}^{n}$. Here we assume that there exists constants $1<\alpha<\infty$, $0<a, b$, such that the measurable functions $A: \mathbf{R}^{n} \times \mathbf{R}^{n} \rightarrow \mathbf{R}^{n}, B: \mathbf{R}^{n} \times \mathbf{R}^{n} \rightarrow \mathbf{R}$ satisfy

$$
\begin{gathered}
|A(x, \xi)| \leq a|\xi|^{\alpha-1}, \\
|B(x, \xi)| \leq b|\xi|^{\alpha-1}, \quad \text { and } \\
\xi \cdot A(x, \xi) \geq|\xi|^{\alpha}
\end{gathered}
$$

for almost all $x \in \Omega$ and all $\xi \in \mathbf{R}^{n}$. By a weak solution to (1.4) we mean a function $u$ locally in the Sobolev class $W_{\alpha}^{1}(\Omega)$ so that

$$
\int_{\Omega}(\nabla \varphi \cdot A+B \varphi) d x=0
$$

for all $\varphi \in C_{0}^{\infty}(\Omega)$. We remark that any such solution is continuous when, if necessary, it is redefined on a set of measure zero [S]. As such, we assume throughout that $u$ is continuous in $\Omega$.

Theorem 1.11 is given in [N2] for the case $n \leq \alpha$. We show here that in fact Theorem 1.11 holds for all $1<\alpha<\infty$. The proof is the same as previously given for the case $n<\alpha$ once we have established Lemma 2.7.

We write $B(x, R)=\left\{y \in \mathbf{R}^{n}|| x-y \mid<R\right\}$ and $|E|$ for the Lebesgue measure of $E \subset \mathbf{R}^{n}$. If $U: E \rightarrow \mathbf{R}^{m}$ is measurable, then we write, for $0<p<\infty$,

$$
\|U\|_{p, E}=\left(\int_{E}|U(x)|^{p} d x\right)^{1 / p} .
$$

If $u$ is a weak solution to (1.4), then we write

$$
D_{u}(x)=|B|^{-1 / \alpha}\|\nabla u\|_{\alpha, B}
$$

where $B=B(x, d(x, \partial \Omega) / 2), d(x, \partial \Omega)=$ distance between $x$ and the boundary of $\Omega, \partial \Omega$.

We need to discuss functions "Lipschitz at the boundary" and write, for $f: \Omega \rightarrow \mathbf{R}^{m}, 0<k \leq 1$,

$$
\|f\|_{\partial}^{k}=\sup _{\substack{x_{1}, x_{2} \in \Omega \\ x_{1} \neq x_{2}}}\left|f\left(x_{1}\right)-f\left(x_{2}\right)\right| /\left(\left|x_{1}-x_{2}\right|^{k}+d\left(x_{1}, \partial \Omega\right)\right)^{k} .
$$

We also need the following local definitions.

$$
\begin{aligned}
\|f\|_{\text {loc }}^{k}= & \sup \left\{\left|f\left(x_{1}\right)-f\left(x_{2}\right)\right| /\left|x_{1}-x_{2}\right|^{k} \mid\right. \\
& \left.x_{1}, x_{2} \in \Omega, x_{1} \neq x_{2},\left|x_{1}-x_{2}\right|<d\left(x_{1}, \partial \Omega\right) / 2\right\},
\end{aligned}
$$




$$
\begin{gathered}
\|f\|_{\text {loc }, \partial}^{k}=\sup \left\{\left|f\left(x_{1}\right)-f\left(x_{2}\right)\right| /\left(\left|x_{1}-x_{2}\right|+d\left(x_{1}, \partial \Omega\right)\right)^{k} \mid\right. \\
\left.x_{1}, x_{2} \in \Omega, x_{1} \neq x_{2},\left|x_{1}-x_{2}\right|<d\left(x_{1}, \partial \Omega\right) / 2\right\} .
\end{gathered}
$$

Clearly,

$$
\|f\|_{\text {loc }, \partial}^{k} \leq \min \left(\|f\|_{\partial}^{k},\|f\|_{\text {loc }}^{k}\right) \leq \max \left(\|f\|_{\partial}^{k},\|f\|_{\text {loc }}^{k}\right) \leq\|f\|^{k} .
$$

The following definition is given in $[\mathrm{L}]$ and with $k=k^{\prime}$, in [GM].

Definition 1.7. For $0<k^{\prime} \leq k \leq 1, \Omega$ is a $\operatorname{Lip}_{k, k^{\prime}}$-extension domain if there is a constant $N$ such that every pair of points $x_{1}, x_{2} \in \Omega$ can be joined by a continuous curve $\gamma \subset \Omega$ for which

$$
\int_{\gamma} d(\gamma(s), \partial \Omega)^{k-1} d s \leq N\left|x_{1}-x_{2}\right|^{k^{\prime}}
$$

The class of $\operatorname{Lip}_{k, k^{\prime}}$-extension domains is wide, including uniform domains and quasiballs, see $[\mathrm{GM}]$ and $[\mathrm{L}]$. When $k^{\prime}<k$ a $\operatorname{Lip}_{k, k^{\prime}}$-extension domain is necessarily bounded $[\mathrm{L}]$ while a $\operatorname{Lip}_{k, k^{\prime}}$-extension domain may be unbounded. See Section 4 for an example.

We use the following facts about these domains.

Lemma 1.8. Suppose that $\Omega$ is a $\operatorname{Lip}_{k, k^{\prime}}$-extension domain with constant $N$. There exists a constant $M$, independent of $f: \Omega \rightarrow \mathbf{R}^{m}$, such that

$$
\|f\|^{k^{\prime}} \leq M\|f\|_{\text {loc }}^{k}
$$

and

$$
\|f\|_{\partial}^{k^{\prime}} \leq M\|f\|_{\text {loc }, \partial}^{k} .
$$

Moreover, $M \leq 5\left(N+(2 \operatorname{diam} \Omega)^{k-k^{\prime}}\right)$ and when $k=k^{\prime}, M \leq 5 N$.

For the proof of (1.9) see [GM] and [L]. The proof of (1.10) is similar to the proof given in [N1] for the case $k=k^{\prime}$. We remark that (1.9) actually characterizes $\operatorname{Lip}_{k, k^{\prime}}$-extension domains. See $[\mathrm{GM}]$ and $[\mathrm{L}]$. We now state the main results.

Theorem 1.11. Suppose that $u$ is a weak solution to (1.4) in $\Omega$ and $0<$ $k \leq 1$. If there is a constant $C_{1}$ such that

$$
D_{u}(x) \leq C_{1} d(x, \partial \Omega)^{k-1},
$$

for all $x \in \Omega$, then there is a constant $C_{2}$, depending only on $n, \alpha, a, b, k$ and $C_{1}$, such that

$$
\|u\|_{\mathrm{loc}, \partial}^{k} \leq C_{2} .
$$

Conversely, if (1.13) holds, then (1.12) holds for all $x \in \Omega$ with $C_{1}$ depending only on $n, \alpha, a, b, k$ and $C_{2}$. 
We also have the following global result.

Theorem 1.14. Suppose that $u$ is a weak solution to (1.4) in a $\operatorname{Lip}_{k, k^{\prime}}$ extension domain $\Omega$, with constant $N, 0<k^{\prime} \leq k \leq 1$. If there is a constant $C_{1}$ such that

$$
D_{u}(x) \leq C_{1} d(x, \partial \Omega)^{k-1}
$$

for all $x \in \Omega$, then there is a constant $C_{2}$, depending only on $n, \alpha, a, b, k^{\prime}, k$, $N$ and $C_{1}$, such that

$$
\|u\|_{\partial}^{k^{\prime}} \leq C_{2}
$$

In which case, $u$ extends continuously to the closure of $\Omega, \bar{\Omega}$. Moreover there are constants $C_{3}$, depending only on $n, \alpha, a, b, k^{\prime}, k$ and $C_{2}$, and $\beta$, depending only on $n, \alpha, a$ and $b$, such that (1.16) (and hence (1.15)) is equivalent to

$$
\|u\|^{k^{\prime}} \leq C_{3}
$$

if $k^{\prime} \leq \beta$. Otherwise, (1.16) only implies that

$$
\|u\|^{\beta} \leq C_{3}(\operatorname{diam} \Omega)^{k^{\prime}-\beta} .
$$

When $k^{\prime}=k$ and $\alpha \geq n$, he above results appear in [N2]. The BMO case when $k=0$ is also in [N2].

\section{A preliminary lemma}

We establish Lemma 2.7 for the proof of Theorem 1.11.

Lemma 2.1. If $u$ is a solution to (1.4) in $\Omega, 0<s<\infty$, then there exists a constant $C$, depending only on $n, s, \alpha, a$, and $b$, such that

$$
|u(x)| \leq C|B|^{-1 / s}\|u\|_{s, B}
$$

for all balls $B=B(x, R)$ with $R<d(x, \partial \Omega)$.

By now, Lemma 2.1 is well-known. It can be obtained by combining results in $[\mathrm{S}]$ and $[\mathrm{IN}]$.

Lemma 2.3. Let $u$ be a solution to (1.4) in $\Omega$ and let $0<s<\infty$ and $1<\sigma<\infty$. There exists an exponent $\alpha^{\prime}$, depending only on $n, \alpha, a$, and $b$, with $\alpha<\alpha^{\prime}$ and a constant $C$, depending only on $n, s, \alpha, \sigma, a$, and $b$ such that

$$
\|\nabla u\|_{\alpha^{\prime}, B} \leq C|B|^{\left(s-\alpha^{\prime}\right) / s \alpha^{\prime}}\|\nabla u\|_{s, \sigma B}
$$

for all balls $B$ with $\sigma B<\Omega$. Here $\sigma B$ is the ball with the same center as $B$ and with radius equal to $\sigma$ times that of $B$. 
Lemma 2.3 can be obtained from results in [ME], and [IN].

Lemma 2.5. Let $A \subset \Omega$ with $|A|>0$ and let $u \in L^{p}(\Omega)$ with $1 \leq p<\infty$. Then for each $c \in \mathbf{R}$,

$$
\left\|u-u_{A}\right\|_{p, \Omega} \leq 2\left(\frac{|\Omega|}{|A|}\right)^{1 / p}\|u-c\|_{p, \Omega}
$$

Here $u_{A}$ is the average value, $|A|^{-1} \int_{A} u(x) d x$.

Lemma 2.5 is obtained with elementary calculations. See $[\mathrm{H}]$.

We use the above lemmas to obtain the following result.

Lemma 2.7. Let $u$ be a solution to (1.4) in $\Omega, 0<s<\infty$ and $1<\sigma<\infty$. There exists a constant $C$, depending only on $n, \alpha, s, \sigma, a$, and $b$, such that

$$
\sup _{B} u-\inf _{B} u \leq C|B|^{(s-n) / s n}\|\nabla u\|_{s, \sigma B}
$$

for all balls $B$ with $\sigma B \subset \Omega$.

Proof. Let $x=$ center of $B, r(B)=$ radius of $B$ and $y \in B$. Then letting $B_{2}=B(y,(\sigma-1) r(B))$ we have, using (2.2) with $s=\alpha,(2.6)$ with $p=\alpha$, $A=B_{2}, \Omega=\sigma B$, the Poincaré inequality and (2.4),

$$
\begin{aligned}
\mid u(x)- & u(y)|\leq| u(x)-u_{B_{2}}|+| u(y)-u_{B_{2}} \mid \\
& \leq C_{1}|B|^{-1 / \alpha}\left\|u-u_{B_{2}}\right\|_{\alpha, \sigma B}+C_{2}\left|B_{2}\right|^{-1 / \alpha}\left\|u-u_{B_{2}}\right\|_{\alpha, B_{2}} \\
& \leq C_{3}(\sigma /(\sigma-1))^{n / \alpha}|B|^{-1 / \alpha}\left\|u-u_{\sigma B}\right\|_{\alpha, \sigma B}+C_{2}\left|B_{2}\right|^{-1 / \alpha}\left\|u-u_{B_{2}}\right\|_{\alpha, B_{2}} \\
& \leq C_{4}|B|^{(\alpha-n) / \alpha n}\|\nabla u\|_{\alpha, \sigma B}+C_{4}\left|B_{2}\right|^{(\alpha-n) / \alpha n}\|\nabla u\|_{\alpha, B_{2}} \\
& \leq C_{5}|B|^{(s-n) / s n}\|\nabla u\|_{s, \sigma B} .
\end{aligned}
$$

Hence, if $y, z \in B$, then

$$
|u(y)-u(z)| \leq|u(y)-u(x)|+|u(z)-u(x)| \leq 2 C_{5}|B|^{(s-n) / s n}\|\nabla u\|_{s, \sigma B} .
$$

Remark 2.9. In the case that $n<\alpha$ and $u \in W_{\alpha}^{1}(\Omega)$, Lemma 2.7 is valid whether or not $u$ is a solution to (1.4) even when $\sigma=1$. See [BI]. 


\section{Proof of Theorem 1.11 and Theorem 1.14}

We need the following facts.

Lemma 3.1. Suppose that $f: \Omega \rightarrow \mathbf{R}^{m}, 0<k \leq 1$ and $0<\eta<1$. There exists a constant $C_{1}$, independent of $f$, such that

$$
\left|f\left(x_{1}\right)-f\left(x_{2}\right)\right| \leq C_{1}\left|x_{1}-x_{2}\right|^{k}
$$

for all $x_{1}, x_{2} \in \Omega$ with $\left|x_{1}-x_{2}\right| \leq \eta d\left(x_{1}, \partial \Omega\right)$ if and only if there is a constant $C_{2}$, independent of $f$, such that

$$
\|f\|_{\text {loc }}^{k}<C_{2} .
$$

A similar statement holds for $\|f\|_{\mathrm{loc}, \partial}^{k}$.

A proof of Lemma 3.1 appears in [L].

Lemma 3.2. Suppose $f: \Omega \rightarrow \mathbf{R}^{m}, 0<k \leq 1$ and $0<\eta<1$. There exists a constant $C_{1}$, independent of $f$, such that

$$
\left|f\left(x_{1}\right)-f\left(x_{2}\right)\right| \leq C_{1}\left|x_{1}-x_{2}\right|^{k}
$$

for all $x_{1}, x_{2} \in \Omega$ with $\left|x_{1}-x_{2}\right|=\eta d\left(x_{1}, \partial \Omega\right)$ if and only if there is a constant $C_{2}$, independent of $f$, such that

$$
\|f\|_{\mathrm{loc}, \partial}^{k}<C_{2}
$$

Proof. Assume that $\|f\|_{\text {loc }, \partial}^{k}<C_{2}$. It follows from Lemma 3.1 that there is a constant $C$ such that

$$
\left|f\left(x_{1}\right)-f\left(x_{2}\right)\right| \leq C\left(\left|x_{1}-x_{2}\right|+d\left(x_{1}, \partial \Omega\right)\right)^{k}
$$

for all $x_{1}, x_{2} \in \Omega$ with $\left|x_{1}-x_{2}\right| \leq \eta d\left(x_{1}, \partial \Omega\right)$. Hence if $\left|x_{1}-x_{2}\right|=\eta d\left(x_{1}, \partial \Omega\right)$, then

$$
\left|f\left(x_{1}\right)-f\left(x_{2}\right)\right| \leq C(1+1 / \eta)^{k}\left|x_{1}-x_{2}\right|^{k} .
$$

Conversely, suppose that

$$
|f(x)-f(y)| \leq C_{1}|x-y|^{k}
$$

for all $x, y \in \Omega$ with $|x-y|=\eta d(x, \partial \Omega)$. Fix $x_{1}, x_{2} \in \Omega$ with $\left|x_{1}-x_{2}\right|<$ $\eta d\left(x_{1}, \partial \Omega\right)$. Let $R_{1}=\eta d\left(x_{1}, \partial \Omega\right)$ and $R_{2}=\eta d\left(x_{2}, \partial \Omega\right)$. Then $R_{2} \leq R_{1}+$ 
$\eta\left|x_{1}-x_{2}\right| \leq R_{1}+\left|x_{1}-x_{2}\right|$ and $R_{1}-\left|x_{1}-x_{2}\right| \leq R_{1}-\eta\left|x_{1}-x_{2}\right| \leq R_{2}$. Hence $\partial B\left(x_{1}, R_{1}\right) \cap \partial B\left(x_{2}, R_{2}\right) \neq \emptyset$. Let $x_{3} \in \partial B\left(x_{1}, R_{1}\right) \cap \partial B\left(x_{2}, R_{2}\right)$. We obtain

$$
\begin{aligned}
\left|f\left(x_{1}\right)-f\left(x_{2}\right)\right| & \leq\left|f\left(x_{1}\right)-f\left(x_{3}\right)\right|+\left|f\left(x_{2}\right)-f\left(x_{3}\right)\right| \\
& \leq C_{1}\left(\left|x_{1}-x_{3}\right|^{k}+\left|x_{2}-x_{3}\right|^{k}\right) \\
& \leq C_{1}\left(\eta^{k} d\left(x_{1}, \partial \Omega\right)^{k}+\eta^{k}\left(\left|x_{1}-x_{2}\right|+d\left(x_{1}, \partial \Omega\right)\right)^{k}\right) \\
& \leq C\left(\left|x_{1}-x_{2}\right|+d\left(x_{1}, \partial \Omega\right)\right)^{k} .
\end{aligned}
$$

It then again follows from Lemma 3.1 that $\|f\|_{\text {loc }, \partial}^{k}<\infty$.

We show that (1.12) implies (1.13). In view of Lemma 3.2, we only need to verify (3.3) for some $\eta<1$. Fix $x_{1}$ and $x_{2}$ with $\left|x_{1}-x_{2}\right|=\frac{1}{4} d\left(x_{1}, \partial \Omega\right)$ and let $B=B\left(x_{1}, 2\left|x_{1}-x_{2}\right|\right)$. Using (2.8) and (1.12) we obtain

$$
\left|u\left(x_{1}\right)-u\left(x_{2}\right)\right| \leq C_{1}|B|^{(\alpha-n) / \alpha n}\|\nabla u\|_{\alpha, B}=C_{1}|B|^{1 / n} D_{u}\left(x_{1}\right) \leq C_{2}\left|x_{1}-x_{2}\right|^{k} .
$$

Conversely, we assume (1.13) and use the fact that if $U$ is a solution to (1.4) in $\Omega$, then there is a constant $C_{3}$, depending only on $n, \alpha, a$, and $b$, so that

$$
\|\nabla U\|_{\alpha, B^{\prime}} \leq C_{3}\left|B^{\prime}\right|^{-1 / n}\|U\|_{\alpha, 2 B^{\prime}}
$$

for all balls $B^{\prime}$ with $2 B^{\prime} \subset \Omega$. See [S]. Using (3.4) with $U=u-u\left(x_{1}\right)$ we obtain, with $B$ as above,

$$
\begin{aligned}
D_{u}\left(x_{1}\right) & =|B|^{-1 / \alpha}\|\nabla u\|_{\alpha, 2 B} \leq C_{4}|B|^{-(\alpha+n) / \alpha n}\left\|u-u\left(x_{1}\right)\right\|_{\alpha, 4 B} \\
& \leq C_{5} d\left(x_{1}, \partial \Omega\right)^{k-1} .
\end{aligned}
$$

This completes the proof of Theorem 1.11.

Next it follows from Theorem 1.11 with Lemma 1.8 that (1.15) implies (1.16) in a $\operatorname{Lip}_{k, k^{\prime}}$-extension domain. The last part of Theorem 1.14 follows by applying Lemma 3.5. Its proof appears in [N2].

Lemma 3.5. Let $u$ be a solution to (1.4) in $\Omega$, continuous in $\bar{\Omega}$ and $0<$ $k \leq 1$. There exists a constant $\beta$, depending on $n, \alpha, a$, and $b$, such that if $k \leq \beta$ and if there exists a constant $C_{1}$ such that

$$
\left|u\left(x_{1}\right)-u\left(x_{2}\right)\right| \leq C_{1}\left|x_{1}-x_{2}\right|^{k}
$$

for all $x_{1} \in \Omega$ and $x_{2} \in \partial \Omega$, then

$$
\|u\|_{\Omega}^{k} \leq C_{2}
$$

where $C_{2}$ depends only on $n, \alpha, a, b$, and $C_{1}$. If $\beta<k,(3.6)$ only implies that

$$
\|u\|^{\beta} \leq C_{2}(\operatorname{diam} \Omega)^{k-\beta} .
$$




\section{Examples}

Example 4.1. We give an example to show that at least in the case that $\alpha=n$ the term $d\left(x_{0}, \partial \Omega\right)$ cannot in general be omitted from (1.13). The radial stretching $f(x)=\left(f_{1}, f_{2}, \ldots, f_{n}\right)=x|x|^{\gamma-1}$ where $\gamma=K^{1 /(1-n)}$ is $K$ quasiconformal in $\mathbf{B}^{n}=\left\{x \in \mathbf{R}^{n}|| x \mid<1\right\}$. As such each component $f_{i}$ of $f$ satisfies an equation of the form (1.4) with $B=0$ and

$$
A(x, h)= \begin{cases}J_{f} D f^{-1}\left|\left(D f^{-1}\right)^{t} h\right|^{n-2}\left(D f^{-1}\right)^{t} h, & \text { if } J_{f} \neq 0 \\ |h|^{n-2} h, & \text { if } J_{f}=0 \text { or does not exist. }\end{cases}
$$

Here $D f$ is the derivative of $f$ and $J_{f}$ is the Jacobian determinant. Also, $\alpha=n$ in (1.5). See [GLM], [M]. It is easy to see that there is a constant $C$, depending only on $n$ and $K$, such that

$$
D_{f_{i}}(x) \leq C
$$

for all $x \in \mathbf{B}^{n}$ and all $1 \leq i \leq n$. However $\left\|f_{i}\right\|^{1}=\infty$ since

$$
\begin{aligned}
& f_{i}(0,0, r, 0, \ldots, 0)-f_{i}(0, \ldots, 0)=r^{\gamma} . \\
& \quad i \text { th }
\end{aligned}
$$

In this case $\beta=K^{1 /(1-n)}$ in Lemma 3.5 is sharp. See [N1].

We next give an example in a $\operatorname{Lip}_{k, k^{\prime}}$-extension domain where $k^{\prime} \leq k$.

Example 4.2. Let $0<k^{\prime} \leq k<1, u=r^{k^{\prime}} \cos k^{\prime} \theta$ and $\Omega=\left\{(x, y)|| y \mid<x^{\gamma}\right.$ with $\left.\gamma=\left(1-k^{\prime}\right) /(1-k), 0<x<1\right\}$. Then $\Omega$ is a $\operatorname{Lip}_{k, k^{\prime}}$-extension domain, see [L], and $u$ is harmonic in $\Omega$. Notice that

$$
\|u\|_{\mathrm{loc}}^{k}<\infty
$$

and

$$
D_{u}(x) \leq C d(x, \partial \Omega)^{k-1},
$$

while we only have

$$
\|u\|^{k^{\prime}}<\infty .
$$

A similar situation holds for the function $v=r^{k^{\prime}}$ in $\Omega$. When $k^{\prime}=(\alpha-2) /(\alpha-1)$, $v$ is a solution to the $\alpha$-harmonic equation, $\alpha>2$,

$$
\operatorname{div}\left(|\nabla v|^{\alpha-2} \nabla v\right)=0
$$




\section{References}

[AG] Astala, K., and F.W. Gehring: Quasiconformal analogues of theorems of Koebe and Hardy and Littlewood. - Michigan Math. J. 32, 1985, 99-108.

[BI] Bojarski, B., and T. IwANIEC: Analytical foundations of the theory of quasiconformal mappings in $R^{n}$. - Ann. Acad. Sci. Fenn. Ser. A I Math. 8, 1983, 257-324.

[D] Duren, P.L.: Theory of $H^{p}$-spaces. - Academic Press, 1970.

[G] Gehring, F.W.: The $L^{p}$-integrability of the partial derivatives of a quasiconformal mapping. - Acta Math. 130, 1973, 265-277.

[GM] Gehring, F.W., and O. Martio: Lipschitz classes and quasiconformal mappings. - Ann. Acad. Sci. Fenn. Ser. A I Math. 10, 1985, 203-221.

[GLM] Granlund, S., P. Lindquist, and O. Martio: Conformally invariant variational integrals. - Trans. Amer. Math. Soc. 277, 1983, 43-73.

[HL] Hardy, G.H., and J.E. Littlewood: Some properties of fractional integrals II. - Math. Z. 34, 1932, 403-439.

[H] Hurri, R.: Poincaré domains in $\mathbf{R}^{n}$. - Ann. Acad. Sci. Fenn. Ser. A I Math. Dissertationes $71,1988$.

[IN] IwANIEC, T., and C.A. NoldER: Hardy-Littlewood inequality for quasiregular mappings in certain domains in $R^{n}$. - Ann. Acad. Sci. Fenn. Ser. A I Math. 10, 1985, 267-282.

[L] Lappalainen, V.: $\operatorname{Lip}_{h}$-extension domains. - Ann. Acad. Sci. Fenn. Ser. A I Math. Dissertationes 56, 1985.

[M] Martio, O.: Potential theoretic aspects of non-linear elliptic partial differential equations. - Report 44, University of Jyväskylä, Department of Mathematics, 1989.

[MRV] Martio, O., S. RICKMAN, and J. VÄISÄLÄ: Definitions for quasiregular mappings. Ann. Acad. Sci. Fenn. Ser. A I Math. 448, 1969, 1-40.

[ME] Meyers, N.G., and A. Elcrat: Some results on regularity for solutions of non-linear elliptic systems and quasi-regular functions. - Duke Math. J. 42, 1975, 121-136.

[NP] NÄKKI, R., and B. PALKA: Lipschitz conditions and quasiconformal mappings. - Indiana Univ. Math. J. 31, 1982, 377-401.

[N1] Nolder, C.A.: A quasiregular analogue of a theorem of Hardy and Littlewood. - Trans. Amer. Math. Soc. 331, 1992, 215-226.

[N2] NoldER, C.A.: Hardy-Littlewood theorems for solutions of elliptic equations in divergence form. - Indiana Univ. Math. J. 40, 1991.

[S] SERrin, J.: Local behavior of solutions of quasi-linear equations. - Acta Math. 111, 1964, 247-302.

[V] VuoRinen, M.: Conformal geometry and quasiregular mappings. - Springer-Verlag, 1988. 